\title{
Sine series expansion of associated Legendre functions
}

\author{
Lóránt Földváry
}

Received: 19 July 2014 / Accepted: 17 November 2014 / Published online: 13 January 2015

(C) Akadémiai Kiadó 2014

\begin{abstract}
The most regularly used mathematical tools for representing the geopotential globally are the spherical harmonics, which consists of the longitude-dependent Fourier transform and of the latitude-dependent associated Legendre functions. While the former is by definition a Fourier series, the latter also can be formed to that. An alternative formulation for the sine series expansion of associated Legendre polynomials has been derived based on well-known recurrence formulae. The resulted formulae are subsequently empirically tested for errors to determine the limitations of its use, and strong dependence on the co-latitude has been found.
\end{abstract}

Keywords Sine series expansion · Associated Legendre functions · Spherical harmonics

\section{Introduction}

The most regularly used mathematical tools for representing the geopotential globally are the spherical harmonics, which consists of the longitude-dependent Fourier transform and of the latitude-dependent associated Legendre functions (Heiskanen and Moritz 1967). As this representation is widely used in physical geodesy, it should be noted that spherical harmonics may be represented as 2D-Fourier series as well. The 2D-Fourier series representation of the spherical harmonics gives room for developing different numerically efficient methods for analysis or synthesis of gravity information based on 2D-Fourier transforms (Sneeuw and Bun 1996).

The 2D-Fourier series representation requires a conversion of the associated Legendre functions to a Fourier-series. In practice, associate Legendre polynomials are often considered by the Rodrigues' formula (Dunster 2010), which is characterized by derivatives of the $\sin \vartheta$

L. Földváry $(\bowtie)$

Department of Geodesy and Surveying, Budapest University of Technology and Economics,

Múegyetem rkp 3, H-1111 Budapest, Hungary

e-mail: fl@sci.fgt.bme.hu 
function of order $n+m$, where $n$ is the degree and $m$ is the order of the spherical harmonics and $\vartheta$ is the co-latitude.

The Rodigues' formula is convenient from programming, particularly certain recurrence formulae derived for them, c.f. Eqs. 8.5.1 and 8.5.3 of Abramowitz and Stegun (1965). This representation of the associated Legendre functions, however, overshadows the periodicity by integer multiples of latitude of the certain terms. In fact, associated Legendre functions can be represented as a set of sine series.

There have different solutions for trigonometric expansion of the associated Legendre functions been found in literature, c.f. Eq. 8.7.1. of Abramowitz and Stegun (1965) or Eq. 14.13.1 of Dunster (2010). Some of those formulae are not really efficient for programming purposes due to an infinite summation involved in it. Several solutions have been derived considering programming efficiency mainly with the bloom of the computer era (Hofsommer and Potters 1960; Ricardi and Burrows 1972; Dilts 1985; Elovitz et al. 1989; Swarztrauber 1993; Cheong et al. 2012). These methods deliver useful and efficient solutions, and are to be discussed later on, in Sect. 4. In the present study an alternative solution to those ones is derived, providing sine series expansion of the Legendre functions and of the associated Legendre polynomials.

In the present solution the coefficients of the sine series are presented as linear combination of lower degrees' and/or orders' coefficients. As so, coefficients of higher degrees and orders can be determined conveniently and quickly due to the computationally simple binary operations. The coefficients in this method are presented in closed form.

\section{Deriving the formulation}

Without providing complete and exact definition of the Legendre polynomials and associated Legendre functions, here we start by the definition of the Rodrigues' formula (Heiskanen and Moritz 1967; Dunster 2010):

$$
\begin{aligned}
P_{n}(x) & =\frac{1}{2^{n} n !} \frac{d^{n}}{d x^{n}}\left[\left(x^{2}-1\right)^{n}\right] \\
P_{n, m}(x) & =\frac{(-1)^{m}}{2^{n} n !}\left(1-x^{2}\right)^{m / 2} \frac{d^{n+m}}{d x^{n+m}}\left[\left(x^{2}-1\right)^{n}\right]
\end{aligned}
$$

For more fundamentals on the associated Legendre functions the reader is directed to the "Fundamentals of Potential Theory" chapter of Heiskanen and Moritz (1967) or (more recently) to the "Vector Spherical Harmonics" chapter of Smylie (2013).

In the geodetic practice the argument of Eqs. (1) and (2) is $x=\cos \vartheta=\sin \varphi$. To derive sine transforms analytically, the following trigonometric identities are to be made use:

$$
\begin{aligned}
\sin ^{2}(x) & =\frac{1-\cos (2 x)}{2} \\
\cos ^{2}(x) & =\frac{1+\cos (2 x)}{2} \\
\sin ^{3}(x) & =\frac{3 \sin (x)-\sin (3 x)}{4} \\
\cos ^{3}(x) & =\frac{3 \cos (x)+\cos (3 x)}{4} \\
\cos (x) \cos (y) & =\frac{\cos (x-y)+\cos (x+y)}{2}
\end{aligned}
$$




$$
\begin{aligned}
& \sin (x) \sin (y)=\frac{\cos (x-y)-\cos (x+y)}{2} \\
& \sin (x) \cos (y)=\frac{\sin (x-y)+\sin (x+y)}{2}
\end{aligned}
$$

The following equations present the Legendre polynomials and associated Legendre functions up to degree and order 4 in their most commonly known form. As it is obvious, by making use of Eqs. (3)-(9), their sine transforms can also be determined, which is listed afterwards.

$$
\begin{aligned}
& P_{0,0}(\cos \vartheta)=1 \\
& P_{1,0}(\cos \vartheta)=\cos \vartheta=\sin \left(\vartheta+\frac{\pi}{2}\right) \\
& P_{1,1}(\cos \vartheta)=-\sin \vartheta=-\sin \vartheta \\
& P_{2,0}(\cos \vartheta)=\frac{1}{2}\left(3 \cos ^{2} \vartheta-1\right)=\frac{3}{4} \sin \left(2 \vartheta+\frac{\pi}{2}\right)+\frac{1}{4} \\
& P_{2,1}(\cos \vartheta)=-3 \cos \vartheta \sin \vartheta=-\frac{3}{2} \sin (2 \vartheta) \\
& P_{2,2}(\cos \vartheta)=3 \sin ^{2} \vartheta=-\frac{3}{2} \sin \left(2 \vartheta+\frac{\pi}{2}\right)+\frac{3}{2} \\
& P_{3,0}(\cos \vartheta)=\frac{1}{2}\left(5 \cos ^{3} \vartheta-3 \cos \vartheta\right)=\frac{5}{8} \sin \left(3 \vartheta+\frac{\pi}{2}\right)+\frac{3}{8} \sin \left(\vartheta+\frac{\pi}{2}\right) \\
& P_{3,1}(\cos \vartheta)=-\frac{3}{2}\left(5 \cos ^{2} \vartheta-1\right) \sin \vartheta=-\frac{15}{8} \sin (3 \vartheta)-\frac{3}{8} \sin \vartheta \\
& P_{3,2}(\cos \vartheta)=15 \cos \vartheta \sin ^{2} \vartheta=-\frac{15}{4} \sin \left(3 \vartheta+\frac{\pi}{2}\right)+\frac{15}{4} \sin \left(\vartheta+\frac{\pi}{2}\right) \\
& P_{3,3}(\cos \vartheta)=-15 \sin ^{3} \vartheta=\frac{15}{4} \sin (3 \vartheta)-\frac{45}{4} \sin \vartheta \\
& P_{4,0}(\cos \vartheta)=\frac{1}{8}\left(35 \cos ^{4} \vartheta-30 \cos ^{2} \vartheta+3\right)=\frac{35}{64} \sin \left(4 \vartheta+\frac{\pi}{2}\right) \\
& +\frac{10}{32} \sin \left(2 \vartheta+\frac{\pi}{2}\right)+\frac{9}{64} \\
& P_{4,1}(\cos \vartheta)=-\frac{5}{2}\left(7 \cos ^{3} \vartheta-3 \cos \vartheta\right) \sin \vartheta=-\frac{35}{16} \sin (4 \vartheta)-\frac{5}{8} \sin (2 \vartheta) \\
& P_{4,2}(\cos \vartheta)=\frac{15}{2}\left(7 \cos ^{2} \vartheta-1\right) \sin ^{2} \vartheta=-\frac{105}{16} \sin \left(4 \vartheta+\frac{\pi}{2}\right) \\
& +\frac{15}{4} \sin \left(2 \vartheta+\frac{\pi}{2}\right)+\frac{45}{16} \\
& P_{4,3}(\cos \vartheta)=-105 \cos \vartheta \sin ^{3} \vartheta=\frac{105}{8} \sin (4 \vartheta)-\frac{105}{4} \sin (2 \vartheta) \\
& P_{4,4}(\cos \vartheta)=105 \sin ^{4} \vartheta=\frac{105}{8} \sin \left(4 \vartheta+\frac{\pi}{2}\right)-\frac{105}{2} \sin \left(2 \vartheta+\frac{\pi}{2}\right)+\frac{315}{8}
\end{aligned}
$$

Obviously, for any degree $n$ and order $m$ the associated Legendre function can be re-formed as a set of sine functions, in the following way:

$$
P_{n, m}(\cos \vartheta)=A_{n, m} \sin \left(n \vartheta+j \frac{\pi}{2}\right)+A_{n-2, m} \sin \left((n-2) \vartheta+j \frac{\pi}{2}\right)+\ldots+\text { bias }
$$

where $j= \begin{cases}1, & m \text { even or zero, except } n=0 \\ 0, & m \text { odd, or } n=0\end{cases}$ 
The bias is in the most cases zero, except for the even degree and even order terms.

Sine series of associate Legendre functions of higher degrees and orders can be derived similarly by making use of elementary trigonometric identities. Optionally, the regularly applied recurrence formulae can also be applied; among them, two basic formulae are presented here, Eqs. 8.5.1 and 8.5.3 of Abramowitz and Stegun (1965). The first formula enables deriving a function of a higher degree, based on the functions of the two preceding degrees and the same order.

$$
P_{n+1, m}(\cos \vartheta)=\frac{(2 n+1)}{(n-m+1)} P_{1,0}(\cos \vartheta) P_{n, m}(\cos \vartheta)-\frac{(n+m)}{(n-m+1)} P_{n-1, m}(\cos \vartheta)
$$

A formula for deriving the associated Legendre function of a higher order, based on two neighbouring values with order $m$ reads:

$$
\begin{aligned}
P_{n, m+1}(\cos \vartheta)= & -(n-m) \frac{P_{1,0}(\cos \vartheta)}{P_{1,1}(\cos \vartheta)} P_{n, m}(\cos \vartheta) \\
& +(n+m) \frac{1}{P_{1,1}(\cos \vartheta)} P_{n-1, m}(\cos \vartheta)
\end{aligned}
$$

Finally, an identity to derive sectorials is also made use of. That is

$$
P_{n, n}=(-1)^{n}(2 n-1) ! ! \sin ^{n} \vartheta
$$

In the followings sine series formulae in the form of Eq. (25) are derived making use of Eqs. (26), (27) and (28).

\subsection{Deriving the 'increase degree' formula}

In this step the basic formula for sine series of associated Legendre function with increased degree is derived. The formulation is derived based on Eq. (26) by inserting the sine series forms of the corresponding terms on the right hand side. These three terms are:

$$
\begin{aligned}
P_{n, m}(\cos \vartheta)= & A_{n, m} \sin \left(n \vartheta+j \frac{\pi}{2}\right) \\
& +A_{n-2, m} \sin \left((n-2) \vartheta+j \frac{\pi}{2}\right)+\ldots \\
P_{n-1, m}(\cos \vartheta)= & A_{n-1, m} \sin \left((n-1) \vartheta+j \frac{\pi}{2}\right) \\
& +A_{n-3, m} \sin \left((n-3) \vartheta+j \frac{\pi}{2}\right)+\ldots \\
P_{1,0}(\cos \vartheta)= & \cos \vartheta
\end{aligned}
$$

Inserting these equations to (26), it becomes:

$$
\begin{aligned}
P_{n+1, m}(\cos \vartheta)= & \frac{(2 n+1)}{(n-m+1)} \cos \vartheta\left\{A_{n, m} \sin \left(n \vartheta+j \frac{\pi}{2}\right)\right. \\
& \left.+A_{n-2, m} \sin \left((n-2) \vartheta+j \frac{\pi}{2}\right)+\ldots\right\} \\
& -\frac{(n+m)}{(n-m+1)}\left\{A_{n-1, m} \sin \left((n-1) \vartheta+j \frac{\pi}{2}\right)\right. \\
& \left.+A_{n-3, m} \sin \left((n-3) \vartheta+j \frac{\pi}{2}\right)+\ldots\right\}
\end{aligned}
$$


In the first term of the right hand side of Eq. (32) by the $\cos \vartheta$ is incorporated to the sine terms making use of Eq. (9):

$$
\begin{aligned}
P_{n+1, m}(\cos \vartheta)= & \frac{(2 n+1)}{(n-m+1)}\left\{\frac{A_{n, m}}{2} \sin \left((n+1) \vartheta+j \frac{\pi}{2}\right)\right. \\
& +\frac{A_{n, m}+A_{n-2, m}}{2} \sin \left((n-1) \vartheta+j \frac{\pi}{2}\right) \\
& \left.+\frac{A_{n-2, m}+A_{n-4, m}}{2} \sin \left((n-3) \vartheta+j \frac{\pi}{2}\right)+\ldots\right\} \\
& -\frac{(n+m)}{(n-m+1)}\left\{A_{n-1, m} \sin \left((n-1) \vartheta+j \frac{\pi}{2}\right)\right. \\
& \left.+A_{n-3, m} \sin \left((n-3) \vartheta+j \frac{\pi}{2}\right)+\ldots\right\}
\end{aligned}
$$

By re-arranging the equation to separate the different sine terms we get:

$$
\begin{aligned}
P_{n+1, m}(\cos \vartheta)= & \frac{(2 n+1)}{(n-m+1)} \frac{A_{n, m}}{2} \sin \left((n+1) \vartheta+j \frac{\pi}{2}\right) \\
& +\frac{(2 n+1) \frac{A_{n, m}+A_{n-2, m}}{2}-(n+m) A_{n-1, m}}{(n-m+1)} \sin \left((n-1) \vartheta+j \frac{\pi}{2}\right) \\
& +\frac{(2 n+1) \frac{A_{n-2, m}+A_{n-4, m}}{2}-(n+m) A_{n-3, m}}{(n-m+1)} \sin \left((n-3) \vartheta+j \frac{\pi}{2}\right)+\ldots
\end{aligned}
$$

Introducing new variables for the coefficients of the sine terms in (34), the sine transform of an associated Legendre function of degree $n+1$ is:

$$
\begin{aligned}
P_{n+1, m}(\cos \vartheta)= & B_{n+1, m} \sin \left((n+1) \vartheta+j \frac{\pi}{2}\right)+B_{n-1, m} \sin \left((n-1) \vartheta+j \frac{\pi}{2}\right) \\
& +B_{n-3, m} \sin \left((n-3) \vartheta+j \frac{\pi}{2}\right)+\ldots
\end{aligned}
$$

where

$$
\begin{aligned}
B_{n+1, m} & =\frac{(2 n+1)}{(n-m+1)} \frac{A_{n, m}}{2} \\
B_{n-1, m} & =\frac{(2 n+1)}{(n-m+1)} \frac{A_{n, m}+A_{n-2, m}}{2}-\frac{(n+m)}{(n-m+1)} A_{n-1, m} \\
B_{n-3, m} & =\frac{(2 n+1)}{(n-m+1)} \frac{A_{n-2, m}+A_{n-4, m}}{2}-\frac{(n+m)}{(n-m+1)} A_{n-3, m}
\end{aligned}
$$

etc., where $A_{n, m}, A_{n-2, m}, A_{n-4, m}$, etc. are the coefficients of the $P_{n, m}(\cos \vartheta)$ sine series, while the coefficients of $A_{n-1, m}, A_{n-3, m}$, etc. are coefficients of the $P_{n-1, m}(\cos \vartheta)$ sine series. As this equation is constant for order $m$, the phase does not change. In a generalized form Eqs. (36)-(38) can be written as:

$$
B_{n-2 k+1, m}=\frac{(2 n+1)}{(n-m+1)} \frac{A_{n-2 k+2, m}+A_{n-2 k, m}}{2}-\frac{(n+m)}{(n-m+1)} A_{n-2 k+1, m}
$$

where $k=0,1,2 \ldots, \frac{n}{2}$

Special care is needed at the last term of the trigonometric series of (35), which is for even $n+1$ degrees $B_{0, m} \sin \left(0+j \frac{\pi}{2}\right)$, while for odd $n+1$ degrees it is $B_{1, m} \sin \left(\vartheta+j \frac{\pi}{2}\right)$. It can 
be derived that for the last coefficients the valid formulae for these coefficients read:

$$
\begin{aligned}
B_{0, m} & =\frac{(2 n+1)}{(n-m+1)} \frac{\delta_{(m \text { even })}^{0} A_{1, m}}{2}-\frac{(n+m)}{(n-m+1)} A_{0, m} \\
B_{1, m} & =\frac{(2 n+1)}{(n-m+1)} \frac{A_{2, m}+2 A_{0, m}}{2}-\frac{(n+m)}{(n-m+1)} A_{1, m}
\end{aligned}
$$

where $\delta_{(\text {modd })}^{0}$ is the Kronecker delta, which is 1 for even values of $m$, otherwise 0 . For coding aspects it can be said that $A_{n-2 k, m}$ term in (40) is vanishing (as $n-2 k$ becomes -1 ), while the same term in (41) gets doubled, i.e. $2 A_{0, m}$. Furthermore, the $A_{n-2 k+2, m}$ term in (40) also vanishes for $m$ odd cases.

\subsection{Deriving the 'increase order' formula}

In this step the basic formula for sine series of associated Legendre function with increased order is derived. The formulation is derived based on Eq. (27) by inserting the sine series forms of the corresponding terms on the right hand side. These three terms are:

$$
\begin{aligned}
P_{n, m}(\cos \vartheta) & =A_{n, m} \sin \left(n \vartheta+j \frac{\pi}{2}\right)+A_{n-2, m} \sin \left((n-2) \vartheta+j \frac{\pi}{2}\right)+\ldots \\
P_{n-1, m}(\cos \vartheta) & =A_{n-1, m} \sin \left((n-1) \vartheta+j \frac{\pi}{2}\right)+A_{n-3, m} \sin \left((n-3) \vartheta+j \frac{\pi}{2}\right)+\ldots \\
P_{1,0}(\cos \vartheta) & =\cos \vartheta \\
P_{1,1}(\cos \vartheta) & =-\sin \vartheta
\end{aligned}
$$

Inserting these equations to (27), it becomes:

$$
\begin{aligned}
& P_{n, m+1}(\cos \vartheta)=(n-m) \frac{\cos \vartheta}{\sin \vartheta} \\
& \quad \times\left\{A_{n, m} \sin \left(n \vartheta+j \frac{\pi}{2}\right)+A_{n-2, m} \sin \left((n-2) \vartheta+j \frac{\pi}{2}\right)+\ldots\right\} . \\
& -(n+m) \frac{1}{\sin \vartheta}\left\{A_{n-1, m} \sin \left((n-1) \vartheta+j \frac{\pi}{2}\right)\right. \\
& \left.\quad+A_{n-3, m} \sin \left((n-3) \vartheta+j \frac{\pi}{2}\right)+\ldots\right\}
\end{aligned}
$$

Involving the $\frac{1}{\sin \vartheta}$ inside the parentheses, Eq. (46) reads:

$$
\begin{aligned}
& P_{n, m+1}(\cos \vartheta)=(n-m) \cos \vartheta \\
& \times\left\{\frac{A_{n, m} \sin \left(n \vartheta+j \frac{\pi}{2}\right)}{\sin \vartheta}+\frac{A_{n-2, m} \sin \left((n-2) \vartheta+j \frac{\pi}{2}\right)}{\sin \vartheta}+\ldots\right\} \\
&-(n+m)\left\{\frac{A_{n-1, m} \sin \left((n-1) \vartheta+j \frac{\pi}{2}\right)}{\sin \vartheta}\right. \\
&\left.\quad+\frac{A_{n-3, m} \sin \left((n-3) \vartheta+j \frac{\pi}{2}\right)}{\sin \vartheta}+\ldots\right\}
\end{aligned}
$$


Now let's insert $x=n \vartheta+k \frac{\pi}{2}$ and $y=\vartheta$ variables to identity (9), and use for $\cos (x)=$ $\sin \left(x+\frac{\pi}{2}\right)$. It becomes

$$
\sin \left(n \vartheta+k \frac{\pi}{2}\right) \sin (\vartheta)=\frac{1}{2} \sin \left((n-1) \vartheta+(k+1) \frac{\pi}{2}\right)-\frac{1}{2} \sin \left((n+1) \vartheta+(k+1) \frac{\pi}{2}\right)
$$

By re-arranging, and by introducing $j=k+1$ we get:

$$
2 \sin \left(n \vartheta+(j-1) \frac{\pi}{2}\right)=\frac{\sin \left((n-1) \vartheta+j \frac{\pi}{2}\right)}{\sin (\vartheta)}-\frac{\sin \left((n+1) \vartheta+j \frac{\pi}{2}\right)}{\sin (\vartheta)}
$$

In Eq. (47) the $\frac{1}{\sin \vartheta}$ terms can be incorporated to the sine terms by the use of (49). For the purpose, let's re-formulate Eq. (47) by adding and subtracting identical terms in the following way:

$$
\begin{aligned}
& P_{n, m+1}(\cos \vartheta)=(n-m) \cos \vartheta\left\{A_{n, m} \frac{\sin \left(n \vartheta+j \frac{\pi}{2}\right)}{\sin \vartheta}-A_{n, m} \frac{\sin \left((n-2) \vartheta+j \frac{\pi}{2}\right)}{\sin \vartheta}\right. \\
& +\left(A_{n, m}+A_{n-2, m}\right) \frac{\sin \left((n-2) \vartheta+j \frac{\pi}{2}\right)}{\sin \vartheta} \\
& -\left(A_{n, m}+A_{n-2, m}\right) \frac{\sin \left((n-4) \vartheta+j \frac{\pi}{2}\right)}{\sin \vartheta} \\
& +\left(A_{n, m}+A_{n-2, m}+A_{n-4, m}\right) \frac{\sin \left((n-4) \vartheta+j \frac{\pi}{2}\right)}{\sin \vartheta} \\
& \left.-\left(A_{n, m}+A_{n-2, m}+A_{n-4, m}\right) \frac{\sin \left((n-6) \vartheta+j \frac{\pi}{2}\right)}{\sin \vartheta}+\ldots\right\} \\
& -(n+m)\left\{A_{n-1, m} \frac{\sin \left((n-1) \vartheta+j \frac{\pi}{2}\right)}{\sin \vartheta}-A_{n-1, m} \frac{\sin \left((n-3) \vartheta+j \frac{\pi}{2}\right)}{\sin \vartheta}\right. \\
& +\left(A_{n-1, m}+A_{n-3, m}\right) \frac{\sin \left((n-3) \vartheta+j \frac{\pi}{2}\right)}{\sin \vartheta} \\
& \left.-\left(A_{n-1, m}+A_{n-3, m}\right) \frac{\sin \left((n-5) \vartheta+j \frac{\pi}{2}\right)}{\sin \vartheta}+\ldots\right\}
\end{aligned}
$$

Now (49) can be inserted to (50), it simplifies to:

$$
\begin{aligned}
P_{n, m+1}(\cos \vartheta)=( & -m) \cos \vartheta\left\{2 A_{n, m} \sin \left((n-1) \vartheta+(j-1) \frac{\pi}{2}\right)\right. \\
& +2\left(A_{n, m}+A_{n-2, m}\right) \sin \left((n-3) \vartheta+(j-1) \frac{\pi}{2}\right) \\
& \left.+2\left(A_{n, m}+A_{n-2, m}+A_{n-4, m}\right) \sin \left((n-5) \vartheta+(j-1) \frac{\pi}{2}\right)+\ldots\right\} \\
& -(n+m)\left\{2 A_{n-1, m} \sin \left((n-2) \vartheta+(j-1) \frac{\pi}{2}\right)\right. \\
& \left.+2\left(A_{n-1, m}+A_{n-3, m}\right) \sin \left((n-4) \vartheta+(j-1) \frac{\pi}{2}\right)+\ldots\right\}
\end{aligned}
$$


Next step is the incorporation of the $\cos \vartheta$ term in the first term of the right hand side of Eq. (51). This is done by the use of identity (9), and results in

$$
\begin{aligned}
P_{n, m+1}(\cos \vartheta)=( & -m)\left\{A_{n, m} \sin \left(n \vartheta+(j-1) \frac{\pi}{2}\right)+A_{n, m} \sin \left((n-2) \vartheta+(j-1) \frac{\pi}{2}\right)\right. \\
& +\left(A_{n, m}+A_{n-2, m}\right) \sin \left((n-2) \vartheta+(j-1) \frac{\pi}{2}\right) \\
& +\left(A_{n, m}+A_{n-2, m}\right) \sin \left((n-4) \vartheta+(j-1) \frac{\pi}{2}\right) \\
& +\left(A_{n, m}+A_{n-2, m}+A_{n-4, m}\right) \sin \left((n-4) \vartheta+(j-1) \frac{\pi}{2}\right) \\
& \left.+\left(A_{n, m}+A_{n-2, m}+A_{n-4, m}\right) \sin \left((n-6) \vartheta+(j-1) \frac{\pi}{2}\right)+\ldots\right\} \\
& -(n+m)\left\{2 A_{n-1, m} \sin \left((n-2) \vartheta+(j-1) \frac{\pi}{2}\right)\right. \\
& \left.+2\left(A_{n-1, m}+A_{n-3, m}\right) \sin \left((n-4) \vartheta+(j-1) \frac{\pi}{2}\right)+\ldots\right\}
\end{aligned}
$$

By re-arranging the equation to separate the different sine terms we get:

$$
\begin{aligned}
P_{n, m+1}(\cos \vartheta)= & (n-m) A_{n, m} \sin \left(n \vartheta+(j-1) \frac{\pi}{2}\right) \\
& +\left\{(n-m)\left(2 A_{n, m}+A_{n-2, m}\right)\right. \\
& \left.-(n+m) 2 A_{n-1, m}\right\} \sin \left((n-2) \vartheta+(j-1) \frac{\pi}{2}\right) \\
& +\left\{(n-m)\left(2 A_{n, m}+A_{n-2, m}+A_{n-4, m}\right)\right. \\
& \left.-(n+m) 2\left(A_{n-1, m}+A_{n-3, m}\right)\right\} \sin \left((n-4) \vartheta+(j-1) \frac{\pi}{2}\right)+\ldots
\end{aligned}
$$

Introducing new variables for the coefficients of the sine terms in (34), the sine transform of an associated Legendre function of order $m+1$ is:

$$
\begin{aligned}
P_{n, m+1}(\cos \vartheta)= & B_{n, m} \sin \left(n \vartheta+\tilde{j} \frac{\pi}{2}\right)+B_{n-2, m} \sin \left((n-2) \vartheta+\tilde{j} \frac{\pi}{2}\right) \\
& +B_{n-4, m} \sin \left((n-4) \vartheta+\tilde{j} \frac{\pi}{2}\right)+\ldots+B_{0, m} \sin \left(0+\tilde{j} \frac{\pi}{2}\right)
\end{aligned}
$$

In this case the corresponding coefficients are:

$$
\begin{aligned}
B_{n, m} & =s_{\tilde{j}}(n-m) A_{n, m} \\
B_{n-2, m} & =s_{\tilde{j}}\left[(n-m)\left(2 A_{n, m}+A_{n-2, m}\right)-(n+m) 2 A_{n-1, m}\right] \\
B_{n-4, m} & =s_{\tilde{j}}\left[(n-m)\left(2 A_{n, m}+2 A_{n-2, m}+A_{n-4, m}\right)-(n+m)\left(2 A_{n-1, m}+2 A_{n-3, m}\right)\right]
\end{aligned}
$$

etc., where $A_{n, m}, A_{n-2, m}, A_{n-4, m}$, etc. are the coefficients of the $P_{n, m}(\cos \vartheta)$ sine series, while the coefficients of $A_{n-1, m} A_{n-3, m}$, etc. are coefficients of the $P_{n-1, m}(\cos \vartheta)$ sine series; $\tilde{j}$ is the inverse of $j$, so

$$
\tilde{j}= \begin{cases}1, & \text { if } j=0 \\ 0, & \text { if } j=1\end{cases}
$$

and $s_{\tilde{j}}= \begin{cases}1, & \text { if } j=0 \text { and } \tilde{j}=1 \\ -1, & \text { if } j=1 \text { and } \tilde{j}=0\end{cases}$ 
The generalized form of Eqs. (55)-(57) reads:

$$
B_{n-2 k, m}=2 s_{\tilde{j}}\left[(n-m)\left(\frac{A_{n-2 k, m}}{2}+\sum_{i=0}^{k-1} A_{n-2 i, m}\right)-(n+m) \sum_{i=0}^{k-1} A_{n-2 i-1, m}\right]
$$

where $k=0,1,2 \ldots, \frac{n}{2}$

Similarly to Eqs. (40) and (41), special care is needed at the last term of the trigonometric series of (58), which is for even $n$ degrees $B_{0, m} \sin \left(0+j \frac{\pi}{2}\right)$, while for odd $n$ degrees it is $B_{1, m} \sin \left(\vartheta+j \frac{\pi}{2}\right)$. For the sectorial and near-sectorial Legendre functions it can be derived that for the last coefficients the valid formulae for these coefficients read:

$$
\begin{aligned}
& B_{1, m}=2 s_{\tilde{j}}\left[(n-m) \frac{-A_{1, m}}{2}-(n+m) \sum_{i=0}^{k-1} A_{n-2 i-1, m}\right] \\
& B_{0, m}=(-1)^{n}(2 n-1) ! ! \frac{1}{2^{n}}\left(\begin{array}{l}
n \\
n / 2
\end{array}\right)
\end{aligned}
$$

In Eq. (60) the symbol !! refers to double factorials. According to Eqs. (10)-(24), only the even degree and even order functions are biased, otherwise $B_{0, m}$ is zero. In the present study Eq. (54) is suggested to use only for the sectorial spherical harmonics, i.e. $m=n$, and for near-sectorials, $m=n-1$ orders (for reasoning see Sect. 3 later on). For such cases the bias would affect even sectorials only. As so, the corresponding formula, i.e. Eq. (60) has been derived for even sectorial cases only, by inserting a power-reduction trigonometric identity formula to Eq. (28). The aforementioned identity to reduce power reads:

$$
\begin{aligned}
& \sin ^{n} \vartheta=\frac{2}{2^{n}} \sum_{k=0}^{\frac{n-1}{2}}(-1)\left(\frac{n-1}{2}-k\right)\left(\begin{array}{l}
n \\
k
\end{array}\right) \sin ((n-2 k) \vartheta) \quad \text { for } n=\text { odd } \\
& \sin ^{n} \vartheta=\frac{1}{2^{n}}\left(\begin{array}{l}
n \\
\frac{n}{2}
\end{array}\right)+\frac{2}{2^{n}} \sum_{k=0}^{\frac{n}{2}-1}(-1)^{\left(\frac{n}{2}-k\right)}\left(\begin{array}{l}
n \\
k
\end{array}\right) \sin \left((n-2 k) \vartheta+\frac{\pi}{2}\right) \quad \text { for } n=\text { even }
\end{aligned}
$$

Obviously, only the $n=$ even case is biased, so it is sufficient to insert the bias term of (61) to (28) to determine the bias of the even degree sectorials, resulting in Eq. (60).

\subsection{Normalized formulation}

The basic formulation is presented by Eqs. (35), (39)-(41) and (54), (58)-(60) for associated Legendre polynomials. Most cases in geodesy the associated Legendre polynomials are normalized be the factor (c.f. Eq. 1-73 of Heiskanen and Moritz 1967)

$$
n f(n, m)=\sqrt{\left(2-\delta_{(m=0)}^{0}\right)(2 n+1) \frac{(n-m) !}{(n+m) !}}
$$

Applying the normalization for the formulation derived above, Eqs. (35) and (54) holds for normalized functions as well with altered coefficients. For the normalized case coefficients of (35) becomes: 


$$
\begin{aligned}
& B_{n-2 k+1, m}=\sqrt{\frac{(2 n+1)(2 n+3)}{(n-m+1)(n+m+1)}} \frac{A_{n-2 k+2, m}+A_{n-2 k, m}}{2} \\
& -\sqrt{\frac{(n+m)(n-m)(2 n+3)}{(n+m+1)(n-m+1)(2 n-1)}} A_{n-2 k+1, m} \\
& B_{0, m}=\sqrt{\frac{(2 n+1)(2 n+3)}{(n-m+1)(n+m+1)}} \frac{\delta_{(m \text { even })}^{0} A_{1, m}}{2} \\
& -\sqrt{\frac{(n+m)(n-m)(2 n+3)}{(n+m+1)(n-m+1)(2 n-1)}} A_{0, m} \\
& B_{1, m}=\sqrt{\frac{(2 n+1)(2 n+3)}{(n-m+1)(n+m+1)}} \frac{A_{2, m}+2 A_{0, m}}{2} \\
& -\sqrt{\frac{(n+m)(n-m)(2 n+3)}{(n+m+1)(n-m+1)(2 n-1)}} A_{1, m}
\end{aligned}
$$

replacing Eqs. (39)-(41). Similarly, the normalized form of coefficients of Eq. (54) can be derived as

$$
\begin{aligned}
B_{n-2 k, m} & =2 s_{\tilde{j}}\left[\sqrt{\frac{n-m}{n+m+1}}\left(\frac{A_{n-2 k, m}}{2}+\sum_{i=0}^{k-1} A_{n-2 i, m}\right)\right. \\
& \left.-\sqrt{\frac{(n+m)(2 n+1)}{(n+m+1)(2 n-1)}} \sum_{i=0}^{k-1} A_{n-2 i-1, m}\right] \\
B_{1, m} & =2 s_{\tilde{j}}\left[\sqrt{\frac{n-m}{n+m+1}} \frac{-A_{1, m}}{2}-\sqrt{\frac{(n+m)(2 n+1)}{(n+m+1)(2 n-1)}} \sum_{i=0}^{k-1} A_{n-2 i-1, m}\right] \\
B_{0, m} & =(-1)^{n} \sqrt{2 \frac{(2 n-1) ! !}{(2 n) ! !}} \frac{1}{2^{n}}\left(\begin{array}{l}
n \\
n / 2
\end{array}\right)
\end{aligned}
$$

replacing Eqs. (58)-(60).

\section{Numerical tests}

In this section numerical tests are presented to determine the accuracy performance of the method. The tests are done in MATLAB R2009b, which has a numerical precision of float arithmetic (the machine $\varepsilon$ ) of $2.220446049250313 \times 10^{-16}$.

As it has already been foreshadowed in the preceding section, generally the use of 'increase degree' formula is suggested, and the 'increase order' formula is used only for determining new sectorials and near-sectorials, c.f. Fig. 1. This becomes essential for the following accuracy analysis.

Normalized Legendre functions has been determined up to degree and order 80 in $\vartheta=70^{\circ}$ both by the Rodrigues' formula, Eqs. (1) and (2), and by the normalized solution of the sine series expansion formula, Eqs. (35) and (54). The results are displayed in logarithmic scale in Figs. 2 and 3 in the same arrangement as in Fig. 1. 


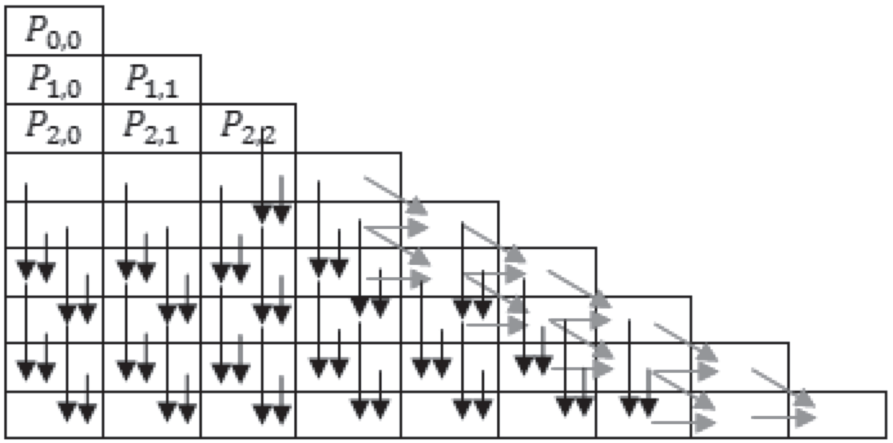

Fig. 1 Scheme of the determining sine series by the recurrence formulae. The black arrow shows the use of 'increase degree' formula, the red arrow refers to the use of 'increase order' formula

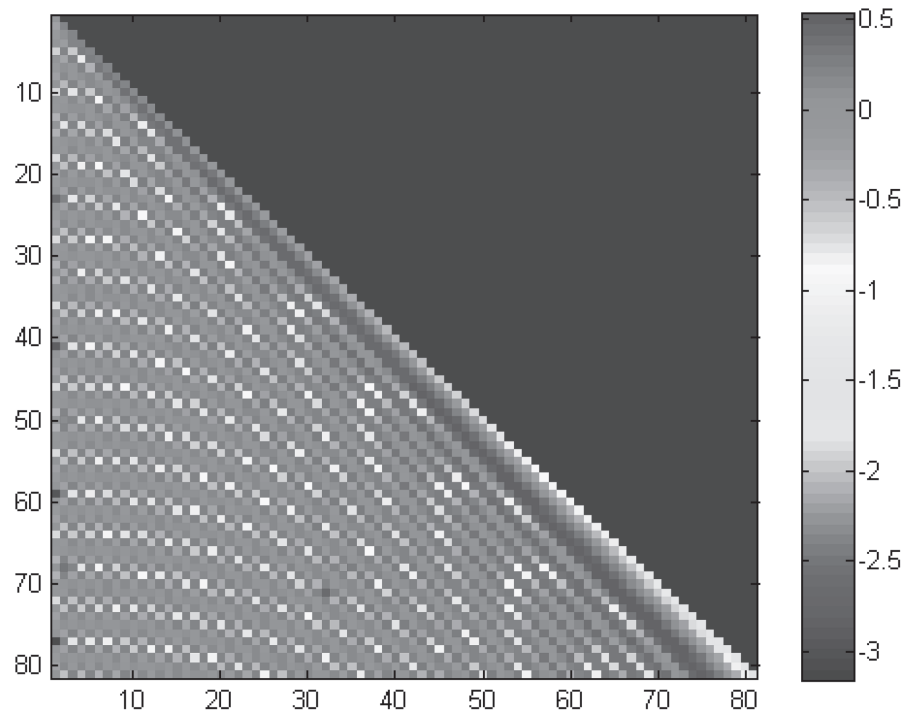

Fig. 2 Associate Legendre functions using the Rodrigues' formulae. The figure is logarithmic, the colorbar displays powers of 10

The similarity is convincingly validates the sine series expansion method. The errors are displayed in Fig. 4. Even though it is negligible at every degree and order, it is systematically increasing by order. Figure 5 shows the signal to noise ratio of the sine series expansion, i.e. the quotient of the signal (at a certain degree and order) and the error (defined as the difference with the value from Rodrigues' formula). Figure 5 reveals even more obvious order-dependence, and that the error is more emphasized at odd orders.

According to Eqs. (27) and (45), the recurrence formula for 'increase order' has a singularity at $\vartheta=0$ degree due to the division by $\sin (\vartheta)$. This is, unavoidably, influences all solutions making use of this equation. In Figs. 6, 7 and 8 the order dependence of the error is investigated by the co-latitude, and test points close to the polar singularity, $\vartheta=1$, at a mid-latitude, $\vartheta=45$, and close to the equator, $\vartheta=89$ were defined. Clearly, close to the polar region the sine series expansion [and also the well-known recurrence formula, i.e. equation (27)] does not work properly apart from the zonal and some near-zonal functions. 


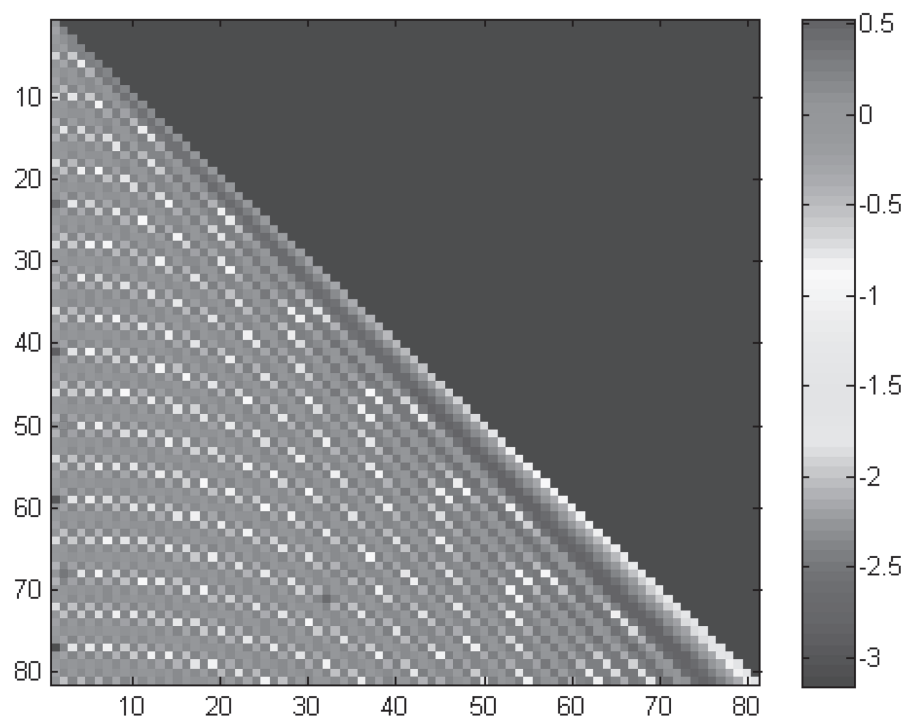

Fig. 3 Associate Legendre functions using the sine series expansion formulae. The figure is logarithmic, the colorbar displays powers of 10.

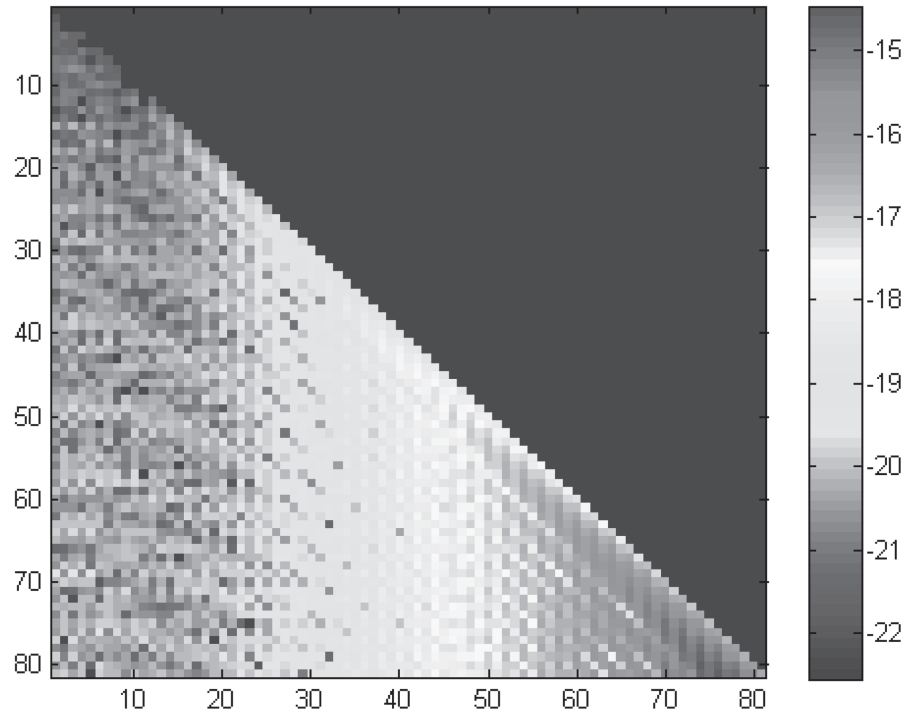

Fig. 4 The error of the sine series expansion, i.e. the difference of Figs. 2 and 3. The figure is logarithmic, the colorbar displays powers of 10

Summarily, the applicability of the sine series is highly dependent on the co-latitude of the point of calculation and of the demanded accuracy. To relate these two criteria to each other, Fig. 9 displays relationship by the error and co-latitude, showing that by co-latitude at which order a certain signal to noise ratio has been exceeded.

According to Fig. 9, the demanded accuracy is essentially depends on the co-latitude. The sine series expansion up to degree and order of 80 safely can be used within the $\vartheta=50-130^{\circ}$ 


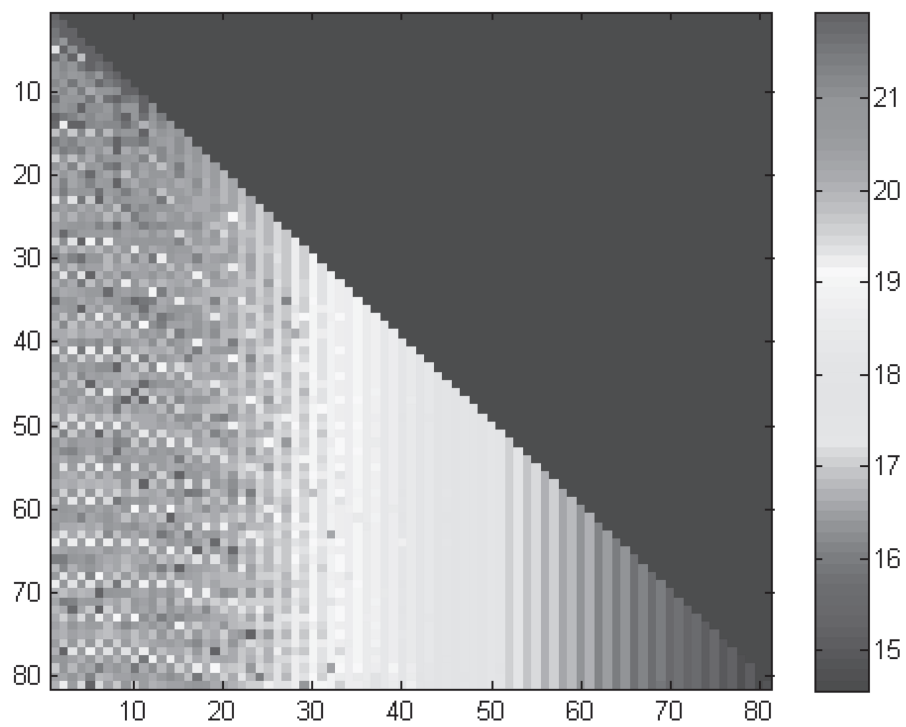

Fig. 5 Signal to noise ratio of sine series expansion, i.e. Fig. 4 divided by Fig. 2. The figure is logarithmic, the colorbar displays powers of 10

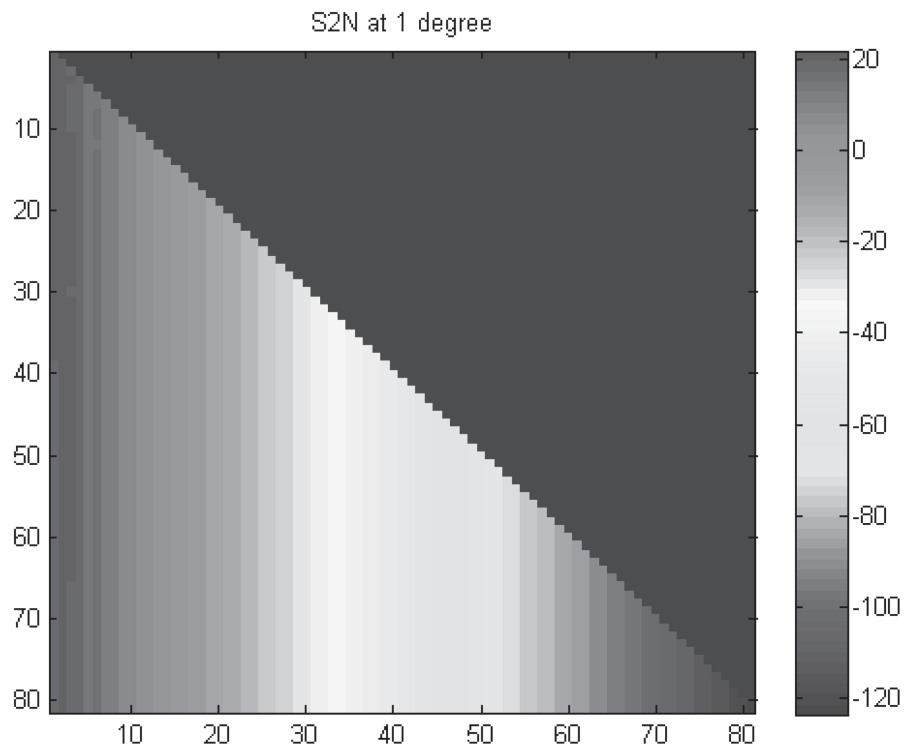

Fig. 6 Signal to noise ratio of sine series expansion at $\vartheta=1$. The figure is logarithmic, the colorbar displays powers of 10

interval, outside of this interval an error estimate should be determined case by case for deciding on its applicability.

A further accuracy test can be defined by investigating the sum of the squared associated Legendre polynomials by the order of the spherical harmonics, $m$. The following equation for normalized polynomials can be derived based on orthogonal properties of the spherical harmonics: 


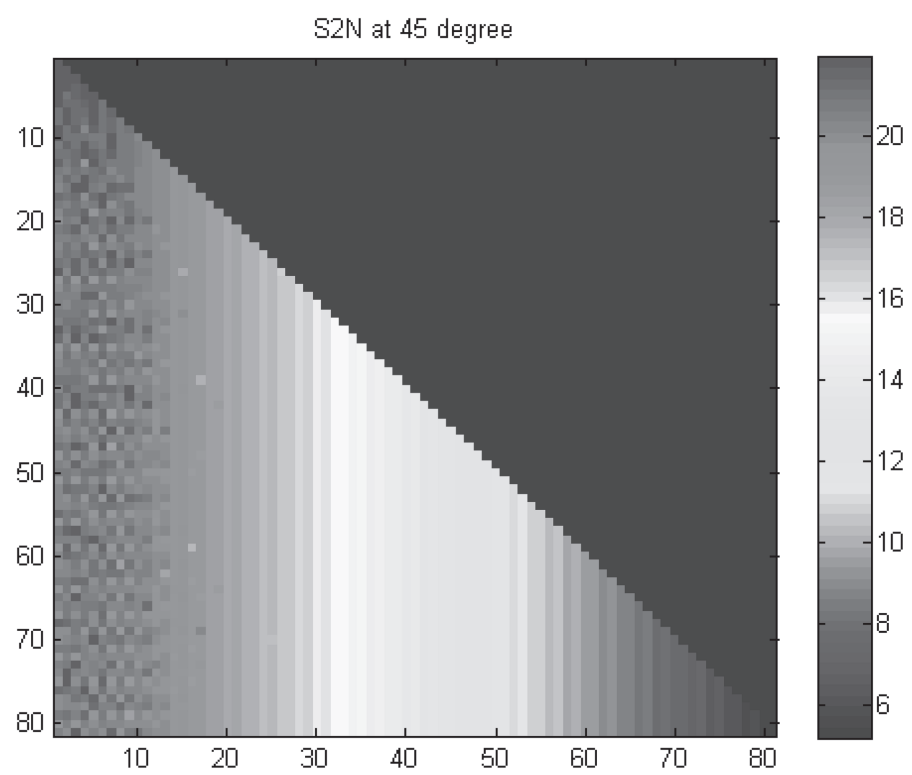

Fig. 7 Signal to noise ratio of sine series expansion at $\vartheta=45$. The figure is logarithmic, the colorbar displays powers of 10

$$
\sum_{m=0}^{n} \bar{P}_{n, m}^{2}(\vartheta)=2 n+1
$$

As the reliability of the method was found to depend on the co-latitude, the accuracy estimate of the method is determined for every $10^{\circ}$ of the co-latitude in the $\left[0^{\circ}, 90^{\circ}\right]$ interval. The results of the corresponding test are displayed on Fig. 10. On the figure absolute value of the deviation from $2 n+1$ can be seen on logarithmic scale.

In Fig. 10 the dependence on the co-latitude is obvious; the accuracy of the derived coefficients is better closer to the equator. By zooming into the low degree part of the deviations, the signal is found to be slightly increasing within the order of magnitude of $10^{-16}$ range, and is not a constant. Also at certain parts the curve is interrupted, which is due to the nonapplicable value for the logarithm of zero. Zero deviation is observed when the difference of $\sum_{m=0}^{n} \bar{P}_{n, m}^{2}$ and $2 n+1$ is below the machine $\varepsilon$ of the software.

\section{Discussion}

In fact, there are solutions for the associated Legendre polynomials (Hofsommer and Potters 1960; Abramowitz and Stegun 1965; Ricardi and Burrows 1972; Dilts 1985; Elovitz et al. 1989; Swarztrauber 1993; Cheong et al. 2012). Regularly presented trigonometric expansion of the associated Legendre functions [c.f. Eq 8.7.1. of Abramowitz and Stegun (1965) or Eq. 14.13.1 of Dunster (2010)] contains infinite summation in the equation. Simple numerical tests have shown that the convergence of the formula is very slow, so restriction to a few terms would be insufficient.

Hofsommer and Potters (1960) has presented first a closed form solution using different identities for deriving associated Legendre polynomials. For 'increase degree' they have applied formulae developed for Legendre functions, i.e. $m=0$ (can be found e.g in 
$52 \mathrm{~N}$ at 89 degree

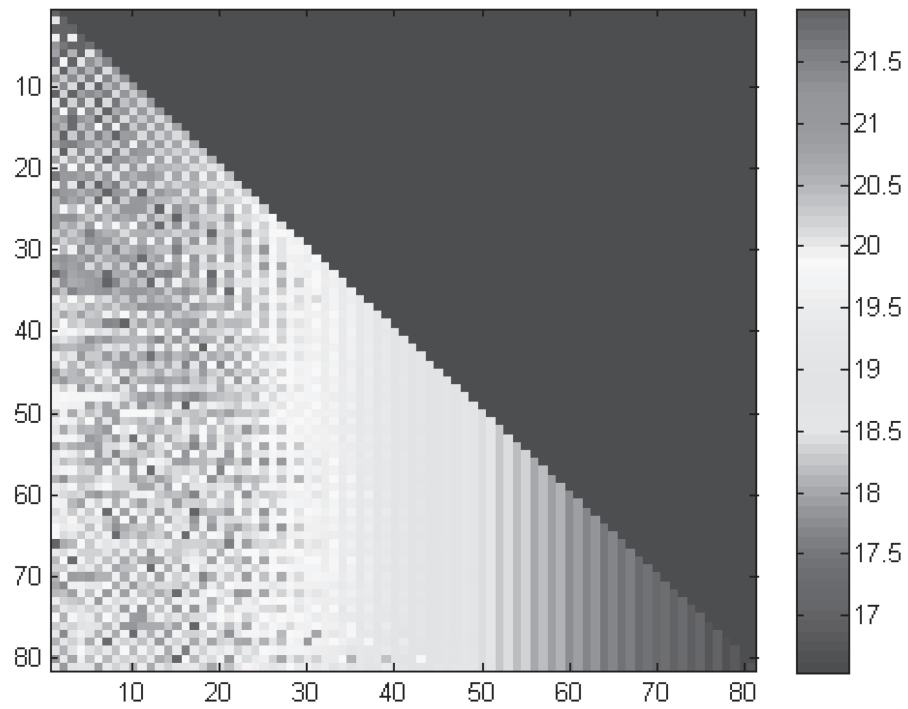

Fig. 8 Signal to noise ratio of sine series expansion at $\vartheta=89$. The figure is logarithmic, the colorbar displays powers of 10

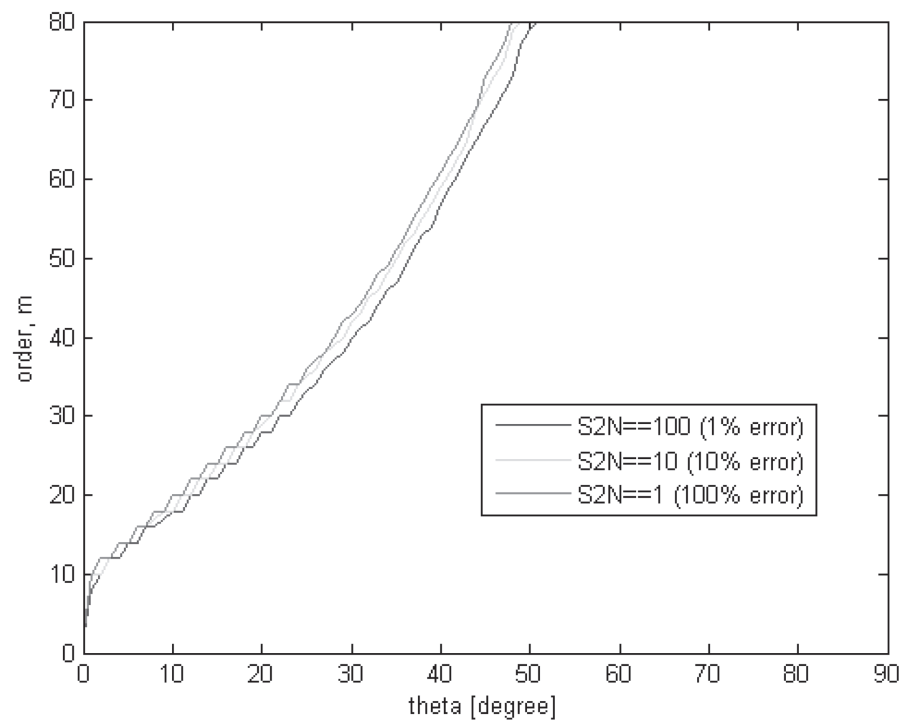

Fig. 9 Signal to noise ratio of sine series expansion as function of order, $m$, and co-latitude $\vartheta$.

Gilbert et al. (1960) or Mughal et al. (2006). As degree is increased for zero order only, the mostly applied formula in their scheme was 'increase order', based on an identity relation $\left[P_{n, m-1} ; P_{n, m}\right] \rightarrow P_{n, m+1}$.

Dilts (1985) developed formulae with complex coefficients using an identity in the form of $\left[P_{n, m-1} ; P_{n, m}\right] \rightarrow P_{n+1, m}$ to increase the degree. Instead of using an 'increase order' formula, Dilts (1985) has used an identity similar to Eq. (28) to determine the sectorials. Dilts (1985) 


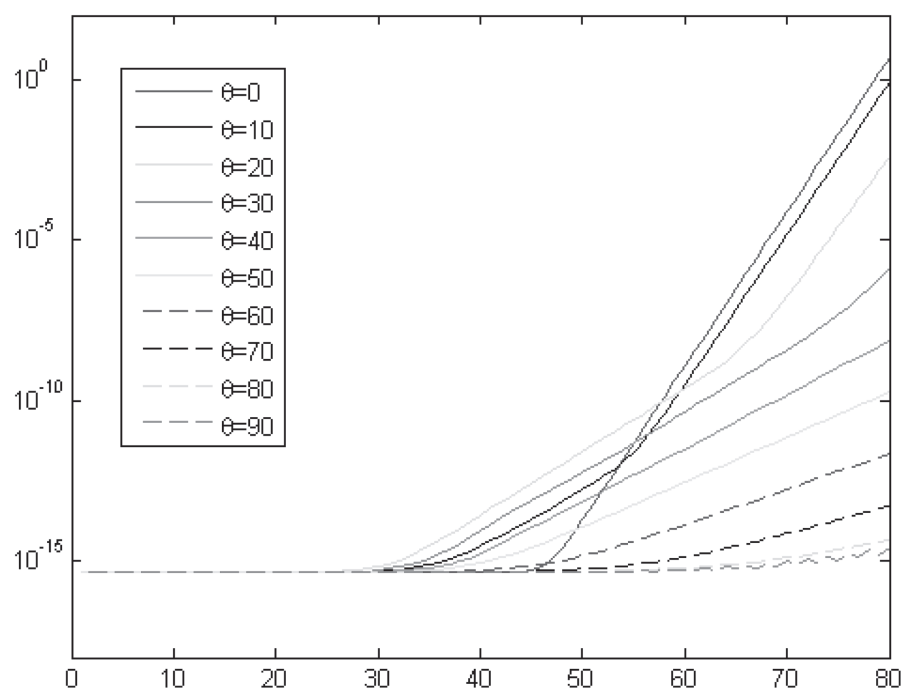

Fig. 10 Difference of the sum of the square of sine series expansion from $2 n+1$ as function of the degree, $n$. The different curves refer to different values of the co-latitude $\vartheta$

has used the classical formula for normalization [c.f Eq. (63)]. (Note that Eq. (63) is the $\sqrt{4 \pi}$ multiple of that, which is the regularly used normalization in geodesy). This way the sine coefficients of the associated Legendre polynomials can be determined under the present precision of the Matlab software up to degree + order $=170$, correspondingly resulting in overflow at high orders of degree 86. This is solved by Elovitz et al. (1989).

The solution scheme of Ricardi and Burrows (1972) [which has also been adopted by Sneeuw and Bun (1996) and partially by Cheong et al. (2012)] is more similar to the present approach: the mostly applied formula is 'increase degree' based on the $\left[P_{n-1, m} ; P_{n, m}\right] \rightarrow P_{n+1, m}$ identity, c.f. Eq. (26). The solution for 'increase order' they use two identities both different from Eq. (27). For sectorials Ricardi and Burrows (1972) use $\left[P_{m, m}\right] \rightarrow P_{m+1, m+1}$, and for near sectorials there's an identity in the form of $\left[P_{m, m}\right] \rightarrow P_{m+1, m}$. For increasing the order, Cheong et al. (2012) uses an identity in the form of $\left[P_{n-2, m-1} ; P_{n, m-1} ; P_{n-2, m+1}\right] \rightarrow P_{n, m+1}$.

The present solution is different in the 'increase order' part, which is derived using an identity $\left[P_{n-1, m} ; P_{n, m}\right] \rightarrow_{n, m+1}^{P}$, c.f. Eq. (27). This formula has been found to be efficient for low latitudes but has singularity at the poles.

In practice, the coding of the linear and of the bias term cannot be done by automatically applying the derived formulation. The applicable terms for that have also been derived here, which has not been discussed in the aforementioned studies.

\section{Summary}

As the present solution the coefficients of the sine series are closed form equations, including linear combination of lower degrees' and/or orders' coefficients, higher degrees and orders can be determined conveniently and quickly due to the computationally simple binary operations (with certain limitations in applicability). The derivation of the present formula was performed analytically without assumptions. Note that the short-coming of these formula- 
tions arises from the original identity formula: as Eq. (27) contains a division by $\sin \vartheta$ on the second term of the right hand side, it has singularity at the poles, which has also influenced the results of the numerical tests. In fact, the choice of the 'increase order' formula is turned out be numerically more unstable. In the presently developed method this formula was therefore highly disregarded against the numerically stable 'increase degree' formula. The sine series expansion for the 'increase order' case has been applied only for the unavoidable sectorial and near-sectorial cases. To improve this shortcoming, further investigations are needed, at the present stage of the research the expansion can be applied with certain considerations taken into account and using for limited bands of the co-latitude. Basically, the closed-form formulation, and the formulation for the linear and the bias terms of the presented method can be considered to be of theoretical importance.

Acknowledgments This study was supported by the OTKA Fund, Project Number K106118.

\section{References}

Abramowitz M, Stegun IA (1965) Handbook of mathematical functions. Dover Publications, New York

Cheong HB, Park JR, Kang HG (2012) Fourier-series representation and projection of spherical harmonic functions. J Geod 86(11):975-990

Dilts GA (1985) Computation of spherical harmonic expansion coefficients via FFT's. J Comput Phys 57(3):439-453

Dunster TM (2010) Legendre and related functions. In: Olver Frank W J, Lozier Daniel M, Boisvert Ronald F, Clark Charles W (eds) NIST handbook of mathematical functions. Cambridge University Press, Cambridge, p 968

Elovitz M, Hill F, Duvall TL (1989) A test of a modified algorithm for computing spherical harmonic coefficients using an FFT. J Comput Phys 80(2):506-511

Gilbert EG, Otterman J, Riordan JF (1960) A tabulation of Fourier transforms of trigonometric functions and Legendre polynomials. Computation Dept., Willow Run Laboratories, University of Michigan, Ann Arbor, Michigan, p 24

Heiskanen WA, Moritz H (1967) Physical geodesy. Freeman and Co., San Francisco, W. H

Hofsommer DJ, Potters ML (1960) Table of Fourier coefficients of associated Legendre functions. Proc KNAW Ser A 63(5):460-480

Mughal AM, Ye X, Iqbal K (2006) Computational algorithm for higher order Legendre polynomial and Gaussian quadrature method. In: Proceedings of the 2006 international conference on scientific computing, Las Vegas, NV, pp 73-77, 26-29 June 2006

Ricardi LJ, Burrows ML (1972) A recurrence technique for expanding a function in spherical harmonics. IEEE Trans Comput 21(6):583-585

Smylie DE (2013) Earth dynamics: deformations and oscillations of the rotating earth. Cambridge University Press, New York

Sneeuw N, Bun R (1996) Global spherical harmonic computation by two-dimensional Fourier methods. J Geod 70:224-232

Swarztrauber PN (1993) The vector harmonic transform method for solving partial differential equations in spherical geometry. Mon Weather Rev 121:3415-3437 